\title{
Low-grade infection due to annular closure device
}

\author{
Nicole Lange $^{1}$ (D) $\cdot$ Bernhard Meyer $^{1} \cdot$ Ehab Shiban $^{1}$
}

Received: 17 June 2018 / Accepted: 25 June 2018 / Published online: 4 July 2018

(C) Springer-Verlag GmbH Austria, part of Springer Nature 2018

Dear Editor,

In response to the letter from Dr. Krutko, we would like to emphasize that bone resorption surrounding a spinal implant is not unique and has been already published in many other series before.

The key message of our case report [1] was the presence of a proven low-grade infection, which is still an underestimated cause of formerly described "aseptic implant failures."

As such, the cases are different. In the case reported by Krutko et al. [2], the patient suffered from these bone changes 1 month following surgery. Therefore, this was probably not a low-grade infection which is usually present with a latency of a few years after surgery. To rule out a low-grade infection, 14-day cultures of implant sonification fluid would have been necessary.

With our case report, we want to raise awareness for lowgrade infections and advocate for the use of 14-day culture from sonification fluid from the removed implant as it is established by the seminal article of Trampusz [3] for hip and knee replacements.

Best regards,

Shiban and Meyer.

\section{References}

1. Lange N, Meyer B, Shiban E (2017) Symptomatic annulus-repairdevice loosening due to a low-grade infection. Acta Neurochir 160: 199-203

2. Krutko AV, Baykov ES, Sadovoy MA (2016) Reoperation after microdiscectomy of lumbar herniation: case report. Int J Surg Case Rep 24:119-123

3. Trampuz A, Piper KE, Jacobson MJ, Hanssen AD, Unni KK, Osmon DR, Mandrekar JN, Cockerill FR, Steckelberg JM, Greenleaf JF, Patel R (2007) Sonication of removed hip and knee prostheses for diagnosis of infection. N Engl J Med 357(7):654-663

This article is part of the Topical Collection on Infection

Nicole Lange

nicole.lange@tum.de

Bernhard Meyer

Bernhard.Meyer@tum.de

Ehab Shiban

Ehab.Shiban@tum.de

1 Department of Neurosurgery, Klinikum rechts der Isar, Technische Universität München, Ismaninger Str. 22, 81675 Munich, Germany 